\title{
Preferred sunspot longitudes: non-axisymmetry and differential rotation
}

\author{
I. G. Usoskin ${ }^{1}$, S. V. Berdyugina ${ }^{2,3}$, and J. Poutanen ${ }^{2}$ \\ ${ }^{1}$ Sodankylä Geophysical Observatory (Oulu unit), 90014 University of Oulu, Finland \\ e-mail: ilya.usoskin@oulu.fi \\ 2 Astronomy Division, PO Box 3000, 90014 University of Oulu, Finland \\ e-mail: juri.poutanen@oulu.fi \\ 3 Institut für Astronomie, ETHZ, 8092 Zürich, Switzerland \\ e-mail: sveta@astro.phys.ethz.ch
}

Received 6 April 2005 / Accepted 15 June 2005

\begin{abstract}
As recently found, the distribution of sunspots is non-axisymmetric and spot group formation implies the existence of two persistent active longitudes separated by $180^{\circ}$. Here we quantitatively study the non-axisymmetry of sunspot occurrence. In a dynamic reference frame inferred from the differential rotation law, the raw sunspot data show a clear clustering around the persistent active longitudes. The differential rotation describing the dynamic frame is quantified in terms of the equatorial angular velocity and the differential rotation rate, which appear to be significantly different from those for individual sunspots. This implies that the active longitudes are not linked to the depth of sunspot anchoring. In order to quantify the observed effect, we introduce a measure of the non-axisymmetry of the sunspot distribution. The non-axisymmetric component is found to be highly significant, and the ratio of its strength to that of the axisymmetric one is roughly $1: 10$. This provides additional constraints for solar dynamo models.
\end{abstract}

Key words. Sun: activity - Sun: magnetic fields - Sun: sunspots

\section{Introduction}

The question whether sunspots appear randomly in longitudes has been a long-standing issue since the early 20th century. Although the existence of preferred longitudes of sunspot formation (active longitudes) has been suggested long ago, the question of their persistency was still a subject of ongoing debates (e.g., Chidambara 1932; Lopez Arroyo 1961; Balthasar \& Schüssler 1983; Vitinsky et al. 1986; Mordvinov $\&$ Kitchatinov 2004). A novel analysis of sunspot group data for the past 120 years revealed the existence of two persistent active longitudes separated by $180^{\circ}$ (Berdyugina \& Usoskin 2003, BU03 henceforth). In BU03 we have shown, using different filtering techniques, that the active longitudes are persistent on a century time scale. An important conclusion of our previous work is that the active longitudes are not fixed in any reference frame (e.g., in the Carrington system), but continuously migrate in longitude with a variable rate. Their migration is defined by changes of the mean latitude of the sunspot formation and the differential rotation. Neglecting this migration results in smearing of the active longitude pattern on time scales of more than one solar cycle. In contrast with the findings of BU03, most previous researchers assumed rotation of the active longitudes with a constant rate, which explains the diversity and contradictions of the previously published results.
The solar active longitudes and their behaviour are very similar to stellar activity phenomena, including the flip-flop cycle detected in binaries and solar-type stars (Berdyugina \& Tuominen 1998; Berdyugina 2004; Berdyugina \& Järvinen 2005). On the Sun the flip-flop phenomenon is observed as the alternation of the major spot activity between the opposite longitudes with a 3.7 year cycle (BU03). The similarity between the sunspot distribution and the activity patterns on cool active stars implies that non-axisymmetry is a fundamental feature of the solar and stellar dynamo mechanisms.

The persistent migrating active longitudes imply the existence of a non-axisymmetric component in the solar dynamo and provide new observational constraints for current solar dynamo models. Therefore, it is important to quantify this effect. As found by BU03, the migration of the active longitudes is defined by the differential rotation and mean latitude of sunspot formation. In the present paper we fit this model to raw sunspot data and determine the differential rotation of the active longitudes. Based on that, we introduce a dynamic reference frame and investigate the distribution of the sunspot area in this new frame. This allows us to account for the migration of the active longitudes. We reveal a double-peaked longitude distribution of the spot area for all sunspots without any filtering and also for the single, strongest spot group observed in each Carrington rotation. More impressively, such a distribution is 
also found for the single, strongest spot group observed in each Carrington rotation. Finally, we introduce a measure of the nonaxisymmetry of the sunspot distribution and estimate a relative strength of the axisymmetric and non-axisymmetric components. The differential rotation law obtained and the measure of the non-axisymmetry can be used to constrain the corresponding dynamo models. Our new analysis confirms the previous conclusions by BU03 on a new basis and dispels the doubts expressed by Pelt et al. (2005) that the active longitude separation is an artefact of the data processing.

In this paper we analyse sunspot group data for the past 120 years. We use daily data on sunspot group locations and areas collected at the Royal Greenwich Observatory, the US Air Force and the National Oceanic and Atmospheric Administration for the years 1878-1996, covering 11 full solar cycles. Here we are primarily interested in the sunspot appearance rather than in their evolution. Accordingly, each spot was included into the analysis only once when it was first mentioned in the database (either on the day of its birth or when it appeared at the East limb), all later records of the spot were ignored. Therefore, in contrast to earlier studies, we analyze only the sunspot emergence. Because of the known asymmetry between the Northern and Southern hemispheres, we investigate them separately. About 40000 sunspots occurred during about 1600 Carrington rotations have been considered in each hemisphere.

\section{A dynamic reference frame}

As suggested in BU03, the migration of the active longitudes on the Sun is defined by the differential rotation and by changes of the mean spot latitude. A standard model of the differential rotation on the Sun relates an angular velocity $\Omega$ with a heliolatitude $\psi$ as follows:

$\Omega=\Omega_{0}-B \sin ^{2} \psi-C \sin ^{4} \psi$,

where $\Omega_{0}$ is the equatorial angular velocity, while $B$ and $C$ describe the differential rotation rate. Here we do not use the higher order term $C \sin ^{4} \psi$, because it influences mostly higher latitudes where sunspots are not observed. Throughout the paper, we use the time step equal to the Carrington period, and Eq. (1) takes the form

$\Omega_{i}=\Omega_{0}-B \sin ^{2}\langle\psi\rangle_{i}$,

where index $i$ denotes the $i$ th Carrington rotation, and $\langle\psi\rangle_{i}$ is the area weighted average latitude of sunspots during this Carrington rotation. Based on that, we introduce a new reference frame which describes the longitudinal migration of active regions with respect to the Carrington frame due to differential rotation:

$\Lambda_{i}=\Lambda_{0}+T_{\mathrm{C}} \sum_{j=N_{0}}^{i}\left(\Omega_{\mathrm{C}}-\Omega_{j}\right)$,

where $\Lambda_{i}$ is the expected active longitude in the $i$ th Carrington rotation, $\Lambda_{0}$ is the location of the active longitude in the $N_{0}$ th Carrington rotation, $T_{\mathrm{C}}=25.38$ days is the sidereal Carrington period and $\Omega_{\mathrm{C}}=360^{\circ} / T_{\mathrm{C}}$. In our model $\Omega_{0}$ and $B$ are the
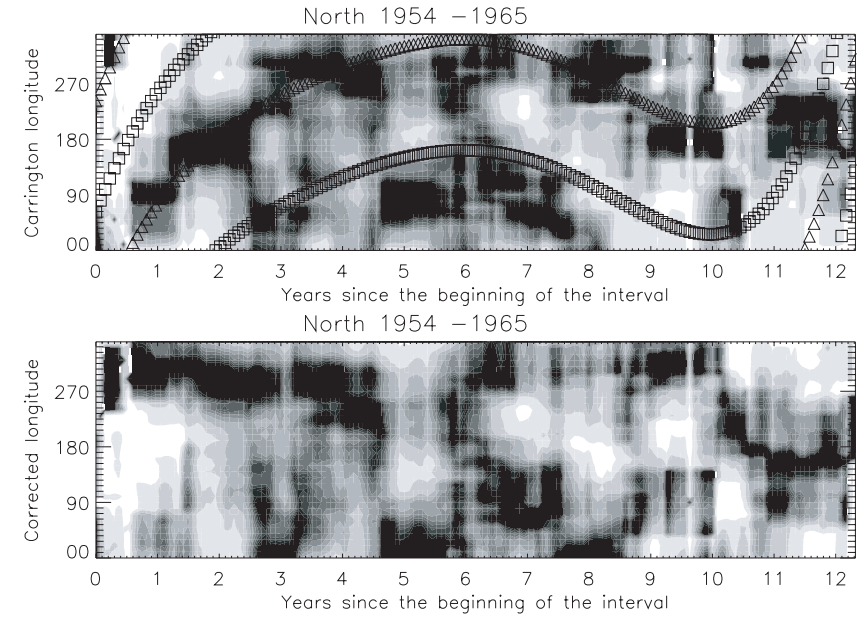

Fig. 1. The longitudinal distribution of the sunspot area during cycle No. 19. The vertical axis denotes the longitude and horizontal axis the time. The upper panel shows the observed Carrington longitudes and the expected migration path of the two active longitudes (shown by squares and triangles) given by Eq. (3) with $B=3.40$ and $\Omega_{0}=$ $14.33 \mathrm{deg} \mathrm{day}^{-1}$. The lower panel shows the same plot but after the longitude correction, i.e. subtraction of the migration path. For better visualization, each plot was filtered using the Lee filter (Lee 1986).

parameters (Eq. (2)) that need to be determined from observations, while $\Lambda_{0}$ is just a constant defining a shift in longitude of the new reference frame with respect to the Carrington system in the $N_{0}$ th Carrington rotation.

The model predicts that for the time $T_{\mathrm{C}}$ the Carrington system makes one full rotation, while the new reference frame does $\Omega_{j} / \Omega_{\mathrm{C}}$ rotations. The difference in longitude accumulated between the two systems over many Carrington periods results in migration of the active longitudes, including a number of full rotations (see BU03). In the following, we discard the full rotations and consider only the circular longitude. In the new reference frame, the longitude of a $k$ th spot in the $i$ th Carrington rotation, $\tilde{\lambda}_{k i}$, is found as follows:

$\tilde{\lambda}_{k i}=\lambda_{k i}-\Lambda_{i}-n 360^{\circ}$

where $\lambda_{k i}$ is the longitude in the Carrington frame, and $n$ is defined to keep $\tilde{\lambda}_{k i}$ within the range $\left[0^{\circ} ; 360^{\circ}\right]$. The transformation to the new reference system is illustrated in Fig. 1, where the upper panel shows the observed longitude-vs.-time pattern of sunspot occurrence during the solar cycle 19. The gray-scale of the plot represents the normalized area of spots occupying a given longitude. We note that the cycloid-like shape of the active longitude is a typical signature of the differential rotation. The normalized area of a $k$ th sunspot in the $i$ th Carrington rotation is defined as

$A_{k i}=S_{k i} / \sum_{j} S_{j i}$

where $S$ is the observed area of the spot corrected for the projection effect, and the sum is taken over all spots in the given Carrington rotation. We consider the normalized area in order to suppress the dependence of the spot area on the phase of the solar cycle. Otherwise, the analysis would be dominated by 
large spot groups around cycle maxima, with the loss of information about active longitudes during the times of minima. The expected migration path of the active longitude, calculated according to our model is shown in the upper panel of Fig. 1 by squares. Triangles denote the same active longitude but shifted by $\pm 180^{\circ}$. One can see that the expected migration path follows the observed concentration of sunspots. Accordingly, if we correct the observed Carrington longitudes of sunspots for the expected migration, the active longitudes should be constant in the new coordinate frame. This is shown in the lower panel of Fig. 1. The sunspot area is clustered around the two active longitudes at about $90^{\circ}$ and $270^{\circ}$. One active longitude is generally more active than the other at a given time, and the dominant activity switches between the two active longitudes, indicating a flip-flop phenomenon (cf. BU03).

The separation of the active longitudes in the dynamic reference frame is clearly seen in Fig. 2 which shows histograms of the area weighted sunspot occurrence in the corrected longitude for the whole data set in the Northern hemisphere. The sunspot distribution in the Carrington frame shows no preferred longitudes (Fig. 2a). The same distribution, but in the dynamic system, shows a clear preference to cluster at two corrected longitudes (Fig. 2b). Note that this histogram shows the longitude distribution of actual spot group areas in the dynamic frame (Eq. (4)), without any filtering, smoothing or other processing of the raw data. This is consistent with our earlier result (BU03) that the signature of the migrating active longitudes is totally smeared out in the Carrington system within 1-2 solar cycles, while a careful account for the migration of the active longitudes allows us to reveal their persistence. A more pronounced double-peaked distribution of the sunspot area is obtained when considering not all spots but only the largest spot within each Carrington rotation (Fig. 2c). Because of the flip-flop effect the two active longitudes are clearly revealed. We emphasize that further processing and averaging of the data significantly increase the revealed non-axisymmetry because of the suppression of the axisymmetric part. For instance, semiannual averaging produces a very significant double-peaked distribution (Figs. 2d and 5 in BU03). Very similar histograms are obtained for the Southern hemisphere (Fig. 3). The filtered distributions (panels $\mathrm{c}$ and d) are shown here only for the purpose of visualisation, and further we will deal only with the raw data distribution shown in Figs. $2 b$ and $3 b$.

\section{Differential rotation parameters}

Let us now estimate the parameters of the differential rotation (Eq. (2)) which fit the raw sunspot group data. For this purpose we employ the least mean squares method. First, we define the deviation between the model and the data as

$\epsilon_{k i}=\min \left(\tilde{\lambda}_{k i} ; \tilde{\lambda}_{k i}-180^{\circ}\right)$,

where indices $i$ and $k$ denote the Carrington rotation number and the spot group within this rotation, respectively. The total discrepancy is then defined as

$\mathcal{E}=\frac{1}{N} \sum_{i} \sum_{k} A_{k i} \epsilon_{k i}^{2}$

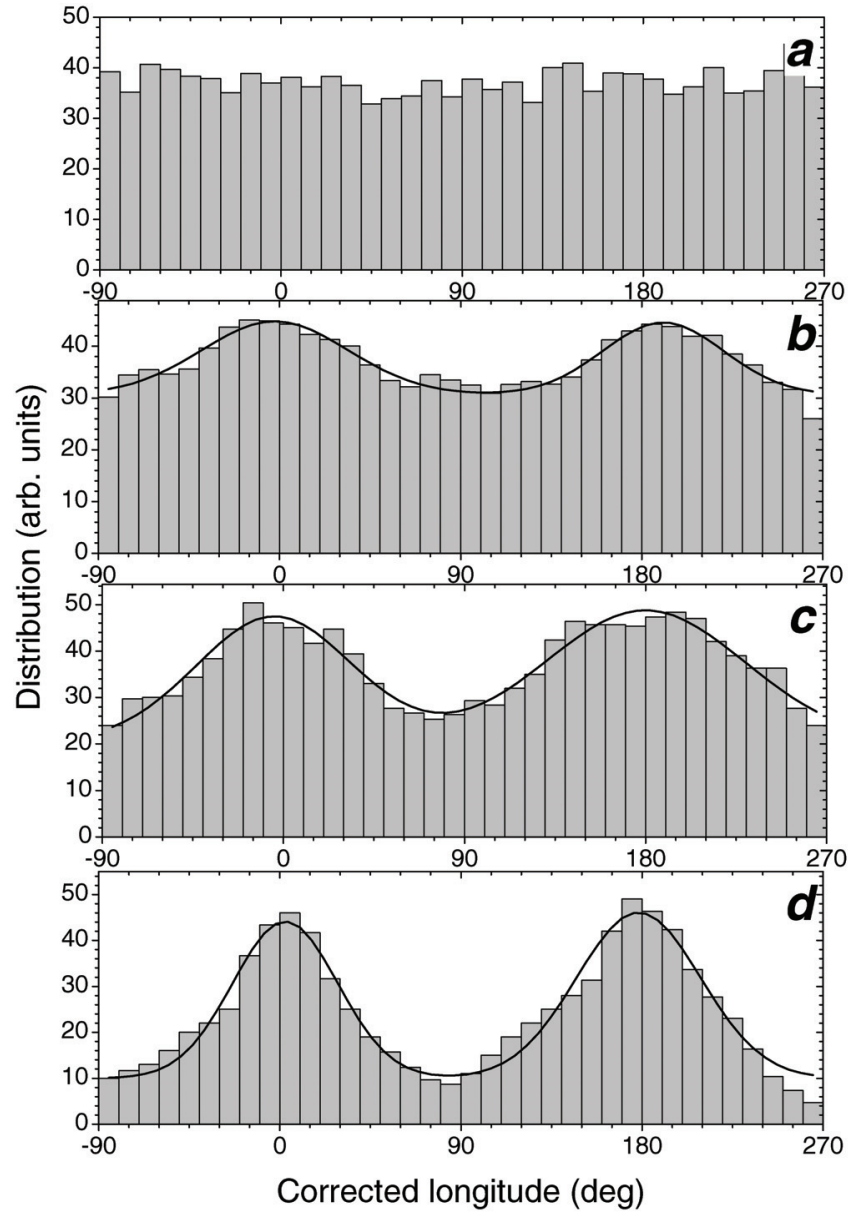

Fig. 2. Longitudinal distributions of sunspot occurrence in the Northern hemisphere for the period 1878-1996. a) Actual sunspots (area weighted) in the Carrington frame. The distribution is nearly isotropic. b) The same as panel a) but in the dynamic reference frame, the non-axisymmetry measure is $\Gamma=0.11$. c) Only the position of one dominant spot for each Carrington rotation is considered, $\Gamma=0.19$. d) The same as in panel c) but for 6-months averages, $\Gamma=0.43$. The solid line depicts the best fit double Gaussian.

where $N=\sum_{i} \sum_{k} A_{k i}$ is the number of Carrington rotations with observed sunspots. Varying the values of $B$ and $\Omega_{0}$ in Eq. (2) we search for a pair which minimizes the discrepancy $\mathcal{E}$. Note that we also vary the value of $\Lambda_{0}$ to obtain the minimum discrepancy.

In order to evaluate the confidence intervals for the bestfitting model parameters, one needs to estimate the uncertainties of the observed data with respect to the fitting model. From the best-fitting parameters corresponding to the $\mathcal{E}_{\min }$ we can build the distribution of the raw data around the expected model (Fig. 2b). This distribution is nearly double Gaussian with the standard deviation of about $\sigma=70^{\circ}$. We adopt this value as a measure of the random scattering of the data with respect to the model. Then we can compute an analogue of $\chi^{2}$ for our model as follows,

$\chi^{2}=\mathcal{E} / \sigma^{2}$.

Note that this is only a scaling of $\mathcal{E}$, i.e. the best-fitting parameters remain the same. The value of $\chi^{2}$ is about 0.5 per formal 


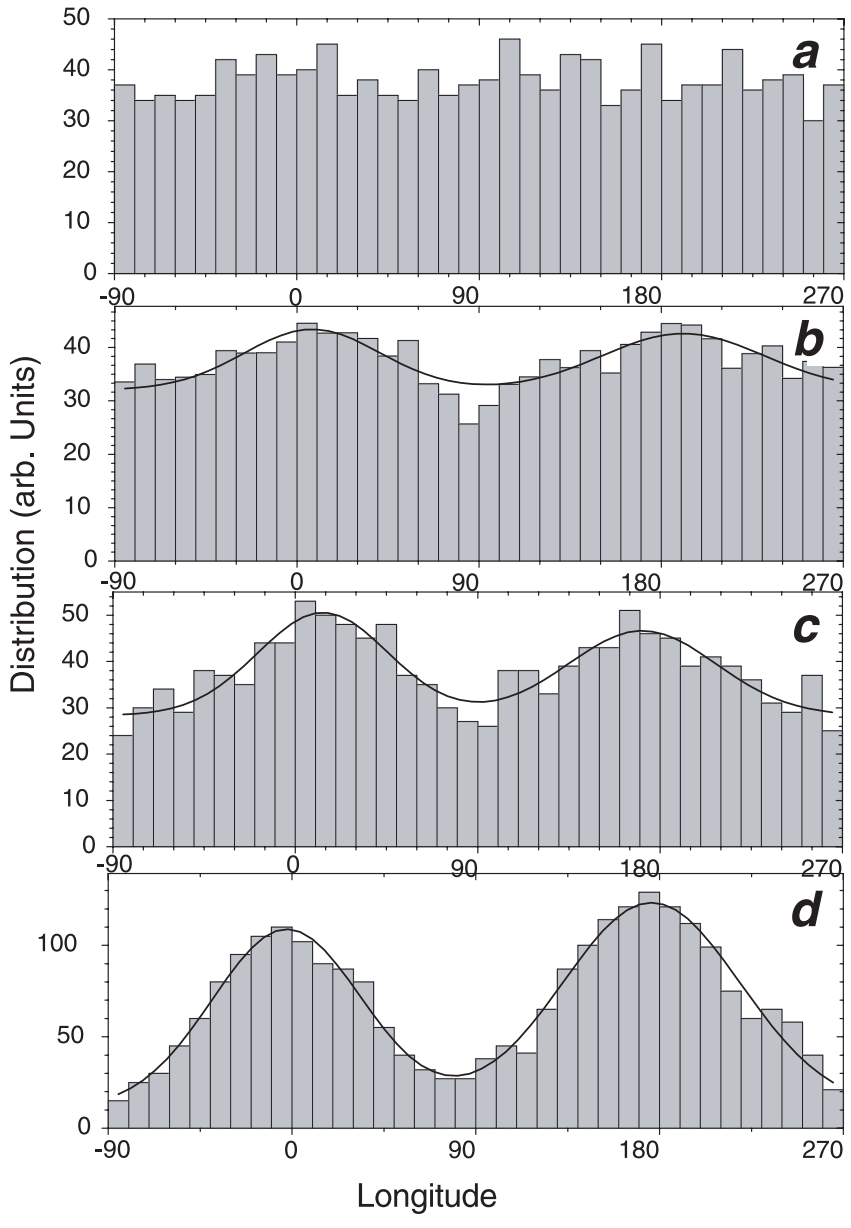

Fig. 3. The same as Fig. 2 but for the Southern hemisphere. The value of the non-axisymmetry $\Gamma$ is $0.09,0.15$ and 0.39 for the panels $\mathbf{b})$, c) and $\mathbf{d})$, respectively.

degree of freedom implying that, although the model fits the data quite well, the data are slightly autocorrelated (i.e. the effective number of degrees of freedom is only half the number of points). The $\chi^{2}$ distribution in the space of the model parameters $B$ and sidereal $\Omega_{0}$ is shown in Fig. 4 .

The best-fitting parameters are $B=3.40 \pm 0.03 \mathrm{deg} \mathrm{day}^{-1}$ and $\Omega_{0}=14.33 \pm 0.01 \mathrm{deg} \mathrm{day}^{-1}$ for the Northern hemisphere, and $B=3.39 \pm 0.02 \mathrm{deg} \mathrm{day}^{-1}$ and $\Omega_{0}=14.31 \pm$ $0.01 \mathrm{deg} \mathrm{day}^{-1}$ for the Southern hemisphere, where the quoted errors correspond to the $90 \%$ confidence interval for one parameter of interest with $\Delta \chi^{2}=\chi^{2}-\min \left(\chi^{2}\right)=2.71$. The results for the North and South being close to each other are nevertheless somewhat different. The difference is formally significant but, taking the uncertainties of the parameter values obtained above as a rough estimate, we can only mention its indicative nature. The distributions in Figs. 2 and 3 in the dynamic reference frame were built using the above best-fitting values of the model parameters. The average value of $B$ is close to that obtained by BU03. We note that the result from BU03 corresponds to a local minimum near $\Omega_{0} \approx 14.2$ and $B=3.46$ deg day $^{-1}$ in Fig. 4 .

When repeating the same procedure for individual cycles, we obtained the best-fitting parameters $B$ varying from 1.5 to $4 \mathrm{deg} \mathrm{day}^{-1}$, and $\Omega_{0}$ from 13.7 to $14.7 \mathrm{deg}_{\text {day }}{ }^{-1}$ (see Fig. 5).

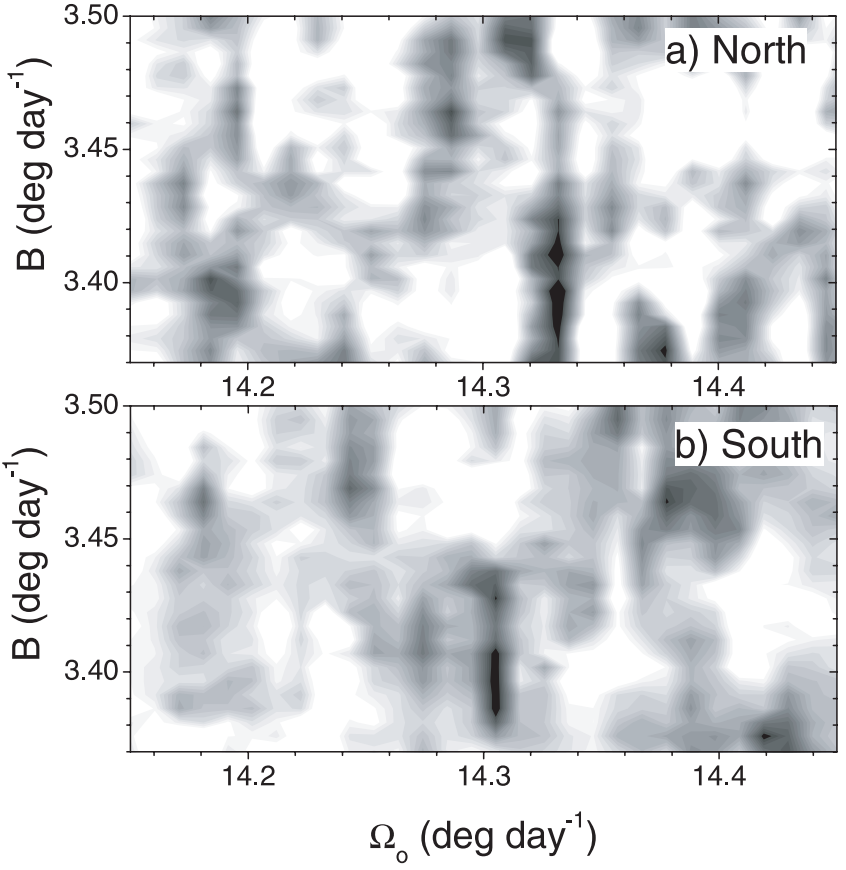

Fig. 4. The distribution of the $\chi^{2}$ values in the space of the model parameters $B$ and sidereal $\Omega_{0}$ (Eq. (2)) for the Northern and Southern hemispheres. The black area depicts the $68 \%$ confidence region $\left(\Delta \chi^{2}=2.3\right)$.

There is a general tendency that smaller $B$ are paired with larger $\Omega_{0}$. Despite the large spread of the best-fitting parameters for individual cycles, the necessity for the differential rotation is apparent, because no good fit can be found for $B=0$. Formal averaging over individual cycles yields the values of $B=3.1 \pm 0.6 \mathrm{deg} \mathrm{day}^{-1}$ and $\Omega_{0}=14.2 \pm 0.24 \mathrm{deg} \mathrm{day}^{-1}$. The analysis for individual cycles could not possibly be used to check the persistence of the active longitudes, because each cycle is fitted independently. However, extending the studied interval not only systematically tightens the allowed area in the parameter space towards the small area determined for the entire data set, as illustrated in Fig. 5, but also proves the persistency of the phenomenon.

In Fig. 6 we compare the present results for the differential rotation of the active longitudes with measurements obtained from sunspots (Balthasar et al. 1986) and surface plasma Doppler shifts (SOHO/MDI, Schou et al. 1998). Note that the SOHO/MDI data were originally approximated by Eq. (1). As seen from Fig. 6, at all latitudes the active longitudes rotate significantly slower than individual sunspots, which is in agreement with the previous finding by BU03. This implies that the active longitudes are not linked to the depth where developed sunspots are anchored. The difference in the rotation of the active longitudes and individual spots is about $0.2 \mathrm{deg} \mathrm{day}^{-1}$ which produces a lag of about 2 full rotations during a solar cycle as reported by BU03. A comparison with the SOHO/MDI measurements is more difficult because of the different shapes of the used approximations. It appears that the active longitudes rotate faster than the local plasma at low latitudes $\left(\psi<18^{\circ}\right)$ and vice-versa at higher latitudes. 


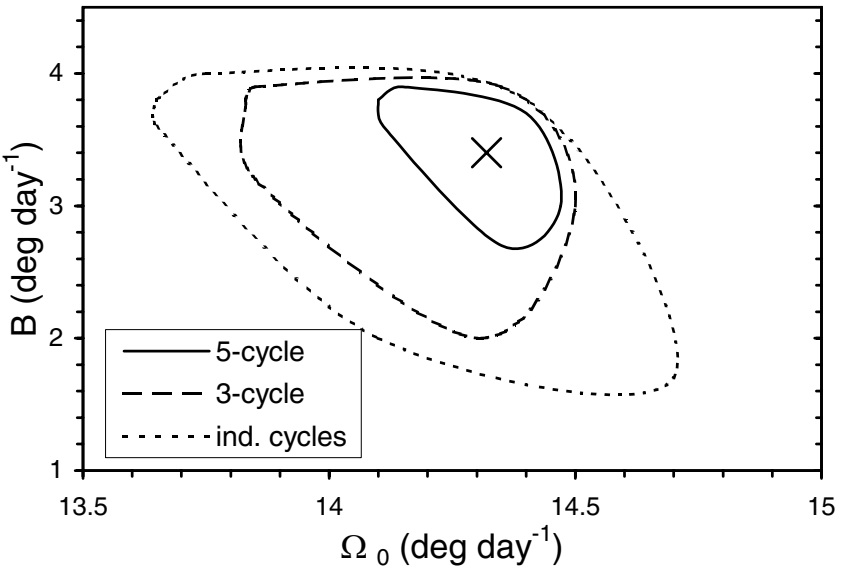

Fig. 5. The area of parameters of the differential rotation in the Northern hemisphere defined using the entire studied interval of 1878-1996 (large cross) as well as from sub-intervals: individual cycles (dotted), 3-cycle intervals (dashed) and 5-cycle intervals (solid).

\section{A measure of the non-axisymmetry}

The existence of the active longitudes implies a long-term asymmetry in the sunspot longitudinal distribution which is related to a non-axisymmetric component of the dynamo mechanism. In order to quantify this we introduce a measure of the non-axisymmetry in the following way. Let us choose the value of $\Lambda_{0}$ so that the two active longitudes correspond to the corrected longitudes of $0^{\circ}$ and $180^{\circ}$ (see Fig. 2b). Depending on the value of the corrected longitude $\tilde{\lambda}_{k i}$, the sunspot with the normalized area $A_{k i}$ contributes to either of the two numbers as follows.

$N_{1}=\sum_{k, i} A_{k i}$, if $\left|\tilde{\lambda}_{k i}\right|<45^{\circ}$ or $\left|\tilde{\lambda}_{k i}-180^{\circ}\right|<45^{\circ}$,

$N_{2}=\sum_{k, i} A_{k i}$, otherwise,

where the summation is taken over all spots in all Carrington rotations. Thus, $N_{1}$ and $N_{2}$ represent the number of spots, weighted by their area, which are close to and far from the active longitudes, respectively. The non-axisymmetry is then defined as

$\Gamma=\frac{N_{1}-N_{2}}{N_{1}+N_{2}}$.

It can take values from 0 to 1 , where 0 corresponds to the axisymmetric sunspot distribution and 1 to the non-axisymmetric distribution.

The non-axisymmetry of the area-weighted sunspot distribution for the entire studied series shown in Figs. $2 b$ and $3 b$ is 0.11 and 0.09 for the Northern and Southern hemispheres, respectively. Without normalizing the spot area (Eq. (5)), the distributions indicate the non-axisymmetry of 0.07 . We conclude, therefore, that the strength of the nonaxisymmetric component is roughly $1: 10$ of that of the axisymmetric one as observed in the sunspot distribution for the last 120 years. For individual cycles $\Gamma$ takes values from 0.07 to 0.3 . We note that at the best-fitting parameters minimizing discrepancy $\mathcal{E}$ (see Fig. 4),

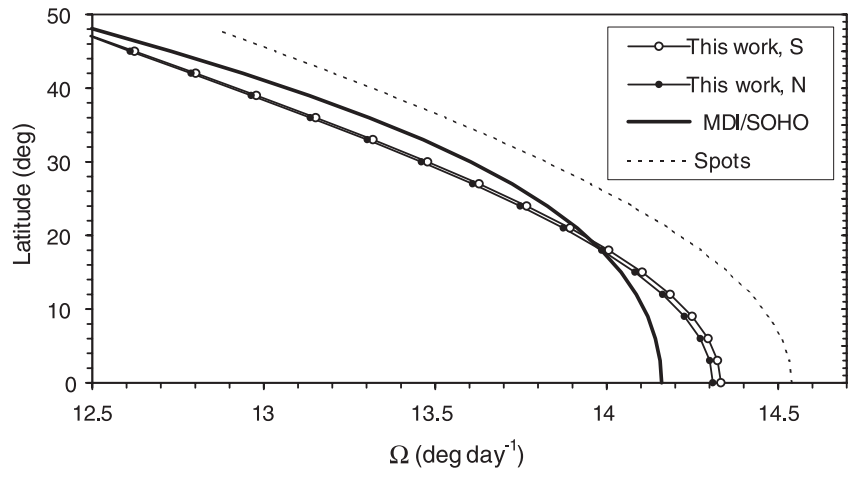

Fig. 6. The sidereal differential rotation of the active longitudes determined in this work compared with that obtained using surface Doppler shifts (SOHO/MDI, Schou et al. 1998) and sunspots (Balthasar et al. 1986).
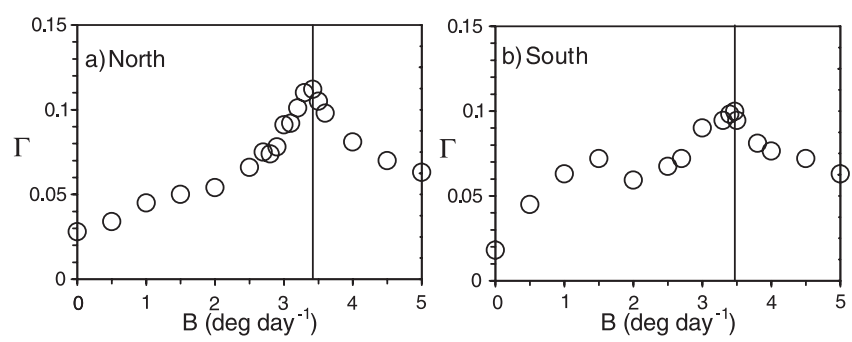

Fig. 7. The measure of the non-axisymmetry $\Gamma$ as a function of the differential rotation parameter $B$ for the Northern (left panel) and Southern (right panel) hemispheres. The values of $B=3.42$ and 3.43 deg day $^{-1}$ as denoted by the vertical lines, correspond to the maximum $\Gamma$ in the Northern and Southern hemispheres, respectively.

$\Gamma$ does not necessarily reach the maximum value, being, however, rather close to it. The dependence of $\Gamma$ on the parameter $B$ is shown in Fig. 7 (parameter $\Omega_{0}$ is now chosen to provide the maximum possible $\Gamma$ ). One can see that the relation has a single peak at about $B=3.42-3.43 \mathrm{deg} \mathrm{day}^{-1}$ (close to the best-fitting value $B=3.39-3.40 \mathrm{deg} \mathrm{day}^{-1}$ minimizing $\mathcal{E}$ ) and decreases when deviating from it. If the longitudes are not corrected for the migration (i.e., $B=0$ ), the non-axisymmetry is $\Gamma=0.02-0.03$ (see Fig. 7a) which is consistent with the nullhypothesis of the axisymmetric distribution (see below). This shows again that neglecting the differential rotation results in complete smearing of the pattern.

In order to check the significance of the obtained nonaxisymmetry measure $\Gamma$ we have performed the following test. For each Carrington rotation we have randomly permuted all the sunspots, i.e., a new random Carrington longitude has been ascribed to each actually observed sunspot while keeping its area. Then the value of $\Gamma$ has been calculated as described above. We have computed the non-axisymmetry $\Gamma$ for 5000 sets of such random-phase sunspot occurrence. The distribution of the value of $\Gamma$ shown in Fig. 8 depicts a nearly Poisson distribution. According to this simulation, the hypothesis of rotation of the active longitudes with a fixed rate (giving $\Gamma=0.02-0.03$; see Figs. 2a, 3a, and 7a,b) cannot be distinguished from the null hypothesis of the axisymmetric sunspot distribution. On the other hand, the probability to obtain $\Gamma=0.11(0.09)$ for an axisymmetric distribution is $<10^{-6}\left(\approx 10^{-5}\right)$ for the Northern 


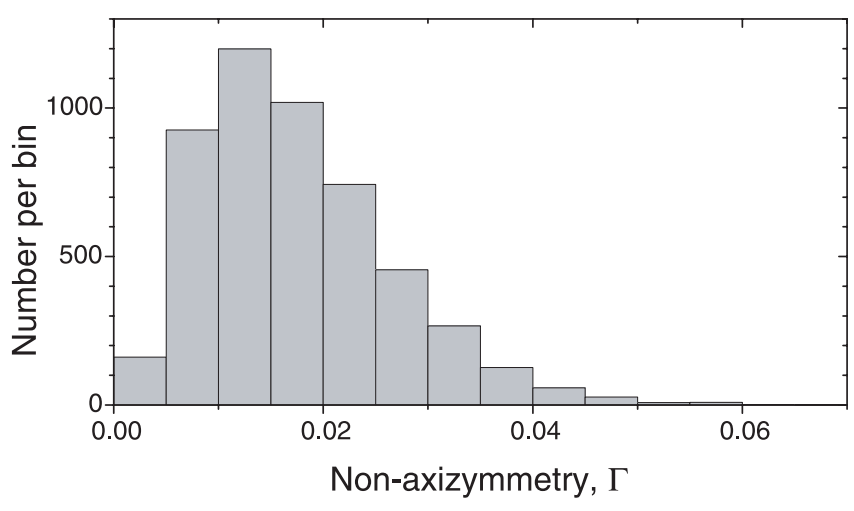

Fig. 8. The distribution of the non-axisymmetry $\Gamma$ calculated for 5000 sets of random-phase sunspot occurrence.

(Southern) hemispheres. This means that the non-axisymmetric component does really exist in the raw data of sunspot occurrence and at the very high significance level. For the case when only the dominant spot is considered (Figs. 2c and 3c) $\Gamma=0.15-0.19$, implying that nearly $60 \%$ of the major spots appear in the vicinity of the active longitudes. Moreover, the non-axisymmetric mode dominates the semiannually averaged sunspot occurrence (Figs. 2d and 3d).

\section{Conclusions}

Active longitudes on the Sun were commonly expected to rotate with a constant rate. This a priori assumption led previous researchers to the conclusion that the active longitudes, if exist, are not stable in their appearance. The recent analysis of the sunspot distribution revealed however that there are two persistent active longitudes which migrate according to the differential rotation law (BU03). In this paper we have confirm this finding and determined the parameters of the differential rotation law affecting the active longitude migration. We emphasize that in the present paper we employ a different approach compared to that used in BU03, where the data were analysed to reveal the underlying regularities. Here we fit a theoretical model of the differential rotation to the raw data without any pre-processing or filtering of the latter. Previously (BU03) we investigated only the non-axisymmetric part of the sunspot distribution while efficiently suppressing the axisymmetric one. This was criticized by Pelt et al. (2005) who claimed that the active longitude pattern found in BU03 is an artefact of the used method. The present analysis, which is based on the raw observed sunspot areas, answers their criticism and confirms that the phenomenon of the persistent active longitudes is real.

The main conclusions obtained in the present paper are listed below.

- The raw sunspot occurrence data for the last 120 years confirm the existence of two preferred longitudes $180^{\circ}$ apart, which migrate in any fixed rotation frame but are persistent throughout the entire period studied in the dynamic frame defined by Eq. (4).
- The differential rotation of the active longitudes is well approximated by Eq. (2) with $B=3.40 \pm 0.03$ (3.39 \pm $0.02)$ deg day ${ }^{-1}$ and sidereal $\Omega_{0}=14.33 \pm 0.01(14.31 \pm$ $0.01) \mathrm{deg} \mathrm{day}^{-1}$ for Northern (Southern) hemispheres, respectively. This is significantly different from the differential rotation of individual spots. This implies that the depth at which sunspots are formed (and affected by the nonaxisymmetric component of the field) is different from that where developed sunspots are anchored.

- The measure of the non-axisymmetry $\Gamma$ (Eq. (10)) for the raw data is found to be $0.11(0.09)$ for the Northern (Southern) hemispheres. These correspond to the significance $<10^{-6}\left(10^{-5}\right)$, respectively. The strength of the nonaxisymmetric dynamo component is roughly $1 / 10$ of that of the axisymmetric one. Appropriate smoothing, filtering or processing of the data suppresses the axisymmetric component and increases the formal measure of the non-axisymmetry.

The above findings imply the existence of a weak, but persistent, non-axisymetric dynamo component with phase migration. The relative strength of this mode and the law of its rotation obtained here provide new constraints for the development of solar dynamo models as was recently undertaken by, e.g., Moss (1999, 2004, 2005).

Acknowledgements. We thank Dmitry Sokoloff and David Moss for stimulating and useful discussions. Solar data have been obtained from the GRO and USAF/NOAA web site http://science.nasa.gov/ ssl/pad/solar/greenwch.htm. The Academy of Finland is acknowledged for the support, grant 43039.

\section{References}

Balthasar, H., \& Schüssler, M. 1983, Sol. Phys., 87, 23

Balthasar, H., Vazquez, M., \& Woehl, H. 1986, A\&A, 155, 87

Berdyugina, S. V. 2004, Sol. Phys., 224, 123

Berdyugina, S. V., \& Järvinen, S. P. 2005, AN, 326, 283

Berdyugina, S. V., \& Tuominen, I. 1998, A\&A, 336, L25

Berdyugina, S. V., \& Usoskin, I. G. 2003, A\&A, 405, 1121 (BU03)

Chidambara, A. 1932, MNRAS, 93, 150

Lee, J. S. 1986, Opt. Engineering, 25(5), 636

Lopez Arroyo, M. 1961, The Observatory, 81, 205

Mordvinov, A. V., \& Kitchatinov, L. L. 2004, Astron. Rep., 48(3), 254

Moss, D. 1999, MNRAS, 306, 300

Moss, D. 2004, MNRAS, 352, L17

Moss, D. 2005, A\&A, 432, 249

Pelt, J., Tuominen, I., \& Brooke, J. 2005, A\&A, 429, 1093

Press, W. H., Teukolsky, S. A., Vetterling, W. T., \& Flannery, B. P. 1992, Numerical Recipes in C (NY, USA: Cambridge Univ. Press) Schou, J., Antia, H., Basu, S., et al. 1998, ApJ, 505, 390

Vitinsky, Yu. I., Kopecky, M., \& Kuklin, G. V. 1986, Statistics of Sunspot Activity (Moscow, USSR: Nauka) 